\title{
Association Between Tumor Mutation Burden (TMB) and immune microenvironment in lung squamous cell carcinoma
}

Dan Yan ( 18367815791@163.com )

Jinhua Municipal Central Hospital https://orcid.org/0000-0002-4336-9153

Yi Chen

The First Affiliated Hospital of Wenzhou Medical University

\section{Research}

Keywords: Tumor Mutation Burden (TMB), Immune microenvironment, Lung squamous cell carcinoma

Posted Date: November 19th, 2020

DOI: https://doi.org/10.21203/rs.3.rs-110577/v1

License: (c) (i) This work is licensed under a Creative Commons Attribution 4.0 International License.

Read Full License 


\section{Abstract}

Background Tumor mutational burden (TMB) is an emerging biomarker for selecting patients with nonsmall cell lung cancer (NSCLC) for immunotherapy. And many studies have revealed high-TMB may be significantly associated with response to PD-1 and PD-L1 blockade immunotherapy.

Methods The RNA-seq transcriptome profiling, simple nucleotide variation and corresponding clinicopathological information of patients with lung squamous cell carcinoma were obtained from The Cancer Genome Atlas (TCGA). We classified samples into the low-TMB group and the high-TMB group based on somatic mutation data from TCGA. KEGG pathways analysis was used to analyze the enriched pathways between two groups. Wilcoxon test was used to analyze the correlation between TMB and clinical pathology. CIBERSORT was used to calculate the immune cell infiltration among different risk groups.

Result Single nucleotide polymorphism (SNP), missense mutation and C > A was most common in patients with lung squamous cell carcinoma. Top 10 most common mutated genes were TTN, TP53, MUC16, CSMD3, RYR2, LRP1B, USH2A, SYNE1, ZFHX4, KMT2D. High-TMB group conferred better oneyear overall survival. KEGG pathways analysis revealed that DEGs were mainly involved in regulation of lymphocyte activation, lymphocyte proliferation, leukocyte proliferation and mononuclear cell proliferation. Infiltration levels of CD8 + T cell, M1 macrophages, follicular helper T cells and activated NK cells in high-TMB group were higher than that in low-TMB group.

Conclusion High TMB correlated with positive one-year survival outcomes. With the development of tumor, TMB increased gradually. TMB might influence the immune infiltrates on patients with lung squamous cell carcinoma.

\section{Background}

Lung cancer is the most frequent cancer ( $11.6 \%$ of the total cases) and one of the leading causes of cancerous deaths worldwide(1). Non-small cell lung cancer (NSCLC) accounts for more than $85 \%$ of all lung cancer cases, and among NSCLC, adenocarcinoma is the most common (accounting for about $40 \%$ of all lung cancers), followed by squamous cell carcinoma (accounting for about $30 \%$ of all lung cancers) (2).

Almost all tumors could be considered potentially immunogenic, the host can generate an immune response, recognize and eradicate tumor cells(3). Tumor-infiltrating immune cells can influence prognosis of tumor and response to immunotherapies $(4,5)$.

Over the decades years, immune checkpoint blockade (ICB) has emerged as a novel effective therapeutic strategy in lung cancer, such as PD-1 inhibitors and PDL-1 inhibitors. Tumor mutation burden (TMB) is defined as the total number of somatic gene coding errors, base substitutions, gene insertion or deletion 
errors detected per million bases. Many studies showed tumor mutational burden (TMB) may be a predictor of immunotherapy response biomarker in tumors, even better than PD-1 expression $(6,7)$.

There is still no comprehensive study explore the associations between TMB and the clinical characteristics and the prognosis of patients with lung squamous cell carcinoma, as well as the relationship between TMB and immune microenvironment. The purpose of this study was to accurately understand the influence of TMB and immune microenvironment in lung squamous cell carcinoma.

\section{Methods}

\section{Data Acquisition}

The RNA-seq transcriptome profiling, simple nucleotide variation and corresponding clinicopathological information of patients with lung squamous cell carcinoma were obtained from The Cancer Genome Atlas (TCGA, https://portal.gdc.cancer.gov/). R package was used to perform the visualization process. All the data in this study were collected from public databases, there was no ethical conflict to declare.

\section{Calculation of TMB and visualization analysis of mutation profiling}

We calculated the TMB score for each sample via 'maftools' R package. And according to the median TMB score, patients with lung squamous cell carcinoma were divided into high-TMB and low-TMB groups. Kaplan-Meier method was conducted to analyze and compare the difference in one-year survival between the two groups. The relationship between TMB score and clinical characteristics (gender, grade, TMN stage) of patients with lung squamous cell carcinoma was analyzed by Wilcoxon test.

\section{Differentially expressed genes and enrichment analysis}

Differentially expressed genes (DEGs) in high-TMB and low-TMB groups were identified by "limma" package with |Log2 fold change $(F C) \mid>1.0$ and False Discovery Rate $(F D R)<0.05$. KEGG was performed to analyze the signal path of DEGs between two groups, and Gene Set Enrichment Analysis (GSEA) was used to analyze the impact of TMB on the prognosis of patients with lung squamous cell carcinoma. NOM P-value $<0.05$ was considered to be significantly enriched and meaningful.

\section{The correlation between immune cells infiltration and TMB levels}

We used the CIBERSORT algorithm to estimate the level of immune cell infiltration in lung squamous cell carcinoma patients, and analyzed the relationship between TMB levels and infiltration of immune cells ( $B$ cells, CD4 + T cells, CD8 + T cells, neutrophils, macrophages, and dendritic cells).

\section{Results}

Landscape of mutation profiles in lung squamous cell carcinoma 
We downloaded the somatic mutation profiling, RNA-seq transcriptome profiling and corresponding clinicopathological information of 491 patients with lung squamous cell carcinoma from TCGA. Missense mutation accounts for the most fraction in all types of mutation (Fig. 1), and single nucleotide polymorphism (SNP) is more common than insertion or deletion in lung squamous cell carcinoma (Fig. 1). What's more, $C>T$ and $C>A$ were most common in single nucleotide variants (SNV) (Fig. 1). Top 10 most common mutated genes were TTN(68\%), TP53(77\%), MUC16(36\%区, CSMD3(40\%), RYR2(35\%), LRP1B(30\%), USH2A(30\%), SYNE1(29\%), ZFHX4(26\%), KMT2D(22\%) (Fig. 1). Waterfall plot showed the mutation information and the different mutation types of each gene in each sample (Fig. 2). The association between different mutated genes were shown in Fig. 3, we can intuitively see TTN is cooccurrence with PAPPA2, ZFHX4, MUC16, and CSMD3 is co-occurrence with RYR3, NAV3, USH2A. The mutually exclusive relationships can be saw between PAPPA2 and TP53.

\section{The correlation between TMB and overall survival, clinical characteristics}

We calculated TMB score and classified samples into the low-TMB group and the high-TMB group based on median value. Kaplan-Meier method showed that compared with low-TMB group, patients in high-TMB group revealed better one-year survival outcomes (Fig. 4A). However, high-TMB correlated with advanced pathological stages(Figure 4B囚.

\section{Differentially expressed genes between high- and low- TMB groups and enrichment analysis}

Differentially expressed genes between high- and low- TMB groups can be seen in Table1, such as CYSLTR2, MS4A1, FAM107A, IGLL1. 
Table 1

Differential expressed genes between low

TMB and high TMB groups

\begin{tabular}{|lll|}
\hline gene & logFC & pValue \\
\hline CYSLTR2 & -1.6898991 & 0.00010269 \\
\hline MS4A1 & -1.1441338 & 0.00058734 \\
\hline FAM107A & -1.0200621 & $7.73 E-05$ \\
\hline IGLL1 & -1.7638948 & 0.00533523 \\
\hline LRRC55 & -1.785876 & 0.00055621 \\
\hline MS4A8 & -1.8295919 & 0.00021727 \\
\hline C20orf85 & -1.1821732 & 0.00062144 \\
\hline SELE & -1.2084087 & 0.00472061 \\
\hline TNFSF8 & -1.1427382 & 0.0032476 \\
\hline NR5A1 & 1.9226092 & $7.87 E-06$ \\
\hline CADM3 & -1.2047907 & $5.53 E-05$ \\
\hline FCRL2 & -1.159438 & 0.0008174 \\
\hline BPIFB1 & -1.4510685 & 0.00011556 \\
\hline ADH1B & -1.1416372 & $9.09 E-06$ \\
\hline INHA & 1.85340517 & 0.00362178 \\
\hline SCGB1A1 & -1.1115467 & $1.63 E-05$ \\
\hline PIGR & -1.0412167 & 0.00022015 \\
\hline C1orf189 & -1.1341047 & 0.00026482 \\
\hline WFDC12 & -1.2491682 & 0.00164705 \\
\hline FAM216B & -1.3900118 & $2.55 E-06$ \\
\hline HS3ST4 & -1.504201 & 0.00026443 \\
\hline PIP & -1.0171485 & 0.00026387 \\
\hline CD22 & -1.0772369 & 0.00013723 \\
\hline FCER2 & -1.3194606 & 0.00204346 \\
\hline C2orf40 & -1.2412141 & $3.72 E-05$ \\
\hline CCL19 & -1.073972 & 0.00058595 \\
\hline
\end{tabular}




\begin{tabular}{|lll|}
\hline gene & logFC & pValue \\
\hline TLR10 & -1.0166526 & 0.00302647 \\
\hline C1orf194 & -1.0624677 & 0.00026991 \\
\hline APOA1 & 6.52638412 & 0.00622402 \\
\hline SMIM24 & 1.10145976 & 0.00793992 \\
\hline
\end{tabular}

Then We conducted the KEGG enrichment analysis and the results showed that these DEGs were mainly involved in lymphocyte activation, lymphocyte proliferation, leukocyte proliferation and mononuclear cell proliferation(Figure 5).

GSEA revealed prominent enrichment of signatures related in the regulation of transcription involved in G1/S transition of mitotic cell cycle, DNA replication, cell meiosis cell cycle process, telomere maintenance via semi-conservative replication, and meiotic cell cycle process in the high TMB group (Fig. 6;Table 2). And in the low TMB group, regulation of microglial cell activation, regulation of $B$ cell activation, humoral immune response and leukocyte migration were enriched group(Figure 7; Table 3). 
Table 2

Gene Set Enrichment Analysis (GSEA)in high- TMB groups

\begin{tabular}{|lllll|}
\hline NAME & ES & NES & $\begin{array}{c}\text { NOM p- } \\
\text { value }\end{array}$ & $\begin{array}{c}\text { FDR q- } \\
\text { value }\end{array}$ \\
\hline GO_REGULATION_OF_TRANSCRIPTION_INVOLVED_ & 0.784 & 2.134 & 0.000 & 0.491 \\
IN_G1_S_TRANSITION_OF_MITOTIC_CELL_CYCLE & & & & \\
\hline GO_DNA_REPLICATION_INITIATION & 0.784 & 2.119 & 0.004 & 0.304 \\
\hline GO_MEIOSIS_I_CELL_CYCLE_PROCESS & 0.579 & 2.103 & 0.006 & 0.257 \\
\hline GO_DNA_POLYMERASE_COMPLEX & 0.766 & 2.082 & 0.000 & 0.251 \\
\hline GO_TELOMERE_MAINTENANCE_VIA_SEMI_ & 0.796 & 2.078 & 0.000 & 0.211 \\
CONSERVATIVE_REPLICATION & & & & \\
\hline GO_MEIOTIC_CELL_CYCLE_PROCESS & 0.538 & 2.076 & 0.002 & 0.181 \\
\hline GO_CATALYTIC_ACTIVITY_ACTING_ON_A_TRNA & 0.637 & 2.052 & 0.006 & 0.207 \\
\hline GO_CHAPERONE_COMPLEX & 0.692 & 2.045 & 0.004 & 0.195 \\
\hline GO_EUCHROMATIN & 0.657 & 2.039 & 0.002 & 0.187 \\
\hline GO_REGULATION_OF_CHROMOSOME_SEPARATION & 0.640 & 2.034 & 0.006 & 0.179 \\
\hline GO_ENDONUCLEASE_COMPLEX & 0.683 & 2.034 & 0.000 & 0.163 \\
\hline GO_MEIOTIC_CHROMOSOME_SEGREGATION & 0.579 & 2.030 & 0.004 & 0.155 \\
\hline GO_NCRNA_3_END_PROCESSING & 0.653 & 2.028 & 0.000 & 0.146 \\
\hline GO_RNA_3_END_PROCESSING & 0.606 & 2.019 & 0.006 & 0.151 \\
\hline GO_FEMALE_MEIOTIC_NUCLEAR_DIVISION & 0.660 & 2.017 & 0.004 & 0.145 \\
\hline GO_HISTONE_EXCHANGE & 0.686 & 2.015 & 0.002 & 0.138 \\
\hline GO_CHROMATIN_REMODELING_AT_CENTROMERE & 0.780 & 2.007 & 0.002 & 0.142 \\
\hline GO_MITOTIC_SPINDLE_ASSEMBLY & 0.638 & 2.006 & 0.008 & 0.136 \\
\hline GO_MITOTIC_SPINDLE_ORGANIZATION & 0.598 & 2.006 & 0.008 & 0.130 \\
\hline GO_CHROMOSOME_SEGREGATION & 0.557 & 2.004 & 0.014 & 0.125 \\
\hline
\end{tabular}


Table 3

Gene Set Enrichment Analysis (GSEA)in low- TMB groups

\begin{tabular}{|c|c|c|c|c|}
\hline NAME & ES & NES & $\begin{array}{l}\text { NOM p- } \\
\text { value }\end{array}$ & $\begin{array}{l}\text { FDR q- } \\
\text { value }\end{array}$ \\
\hline GO_TRANSFORMING_GROWTH_FACTOR_BETA_BINDING & -0.842 & -2.267 & 0.000 & 0.126 \\
\hline GO_EXTERNAL_SIDE_OF_PLASMA_MEMBRANE & -0.677 & -2.265 & 0.002 & 0.065 \\
\hline GO_REGULATION_OF_MICROGLIAL_ & -0.783 & -2.256 & 0.000 & 0.052 \\
\hline \multicolumn{5}{|l|}{ CELL_ACTIVATION } \\
\hline GO_POSITIVE_REGULATION_OF_ & -0.751 & -2.245 & 0.000 & 0.046 \\
\hline \multicolumn{5}{|l|}{ B_CELL_ACTIVATION } \\
\hline GO_PEPTIDE_CROSS_LINKING & -0.741 & -2.230 & 0.000 & 0.045 \\
\hline GO_POSITIVE_REGULATION_OF_ & -0.574 & -2.220 & 0.000 & 0.046 \\
\hline \multicolumn{5}{|l|}{ VASCULATURE_DEVELOPMENT } \\
\hline GO_ANTIGEN_BINDING & -0.829 & -2.217 & 0.002 & 0.042 \\
\hline GO_FC_RECEPTOR_MEDIATED_STIMULATORY_ & -0.724 & -2.214 & 0.002 & 0.039 \\
\hline \multicolumn{5}{|l|}{ SIGNALING_PATHWAY } \\
\hline GO_INTEGRIN_BINDING & -0.633 & -2.210 & 0.002 & 0.037 \\
\hline GO_HUMORAL_IMMUNE_RESPONSE & -0.631 & -2.206 & 0.000 & 0.034 \\
\hline GO_LEUKOCYTE_MIGRATION & -0.608 & -2.202 & 0.002 & 0.034 \\
\hline GO_B_CELL_RECEPTOR_SIGNALING_PATHWAY & -0.842 & -2.202 & 0.000 & 0.031 \\
\hline GO_NEGATIVE_REGULATION_OF_SMOOTH_ & -0.593 & -2.195 & 0.000 & 0.033 \\
\hline \multicolumn{5}{|l|}{ MUSCLE_CELL_PROLIFERATION } \\
\hline GO_PHOSPHORUS_OXYGEN_LYASE_ACTIVITY & -0.746 & -2.194 & 0.000 & 0.032 \\
\hline GO_HUMORAL_IMMUNE_RESPONSE_MEDIATED_BY_ & -0.820 & -2.192 & 0.002 & 0.030 \\
\hline \multicolumn{5}{|l|}{ CIRCULATING_IMMUNOGLOBULIN } \\
\hline GO_CELLULAR_EXTRAVASATION & -0.705 & -2.188 & 0.002 & 0.031 \\
\hline GO_MEMBRANE_INVAGINATION & -0.741 & -2.185 & 0.002 & 0.031 \\
\hline GO_REGULATION_OF_B_CELL_ACTIVATION & -0.715 & -2.185 & 0.002 & 0.029 \\
\hline GO_PHAGOCYTOSIS & -0.658 & -2.181 & 0.004 & 0.029 \\
\hline GO_CELL_RECOGNITION & -0.600 & -2.181 & 0.002 & 0.028 \\
\hline
\end{tabular}




\section{Immune cells infiltration between high- and low- TMB groups}

Violin plot showed different immune cells infiltration between high-TMB and low- TMB groups in in lung squamous cell carcinoma. Infiltration levels of CD8 + T cell, M1 macrophages, follicular helper T cells and activated NK cells in high-TMB group were higher than that in low-TMB group. And infiltration levels of plasma cells, activated CD4 + T memory cells, activated NK cells and M0 macrophages were lower in high-TMB group, compared with low-TMB group(Figure 8).

\section{Discussion}

Tumor mutation burden (TMB) is defined as the total number of somatic gene coding errors, base substitutions, gene insertion or deletion errors detected per million bases. TMB may be a potential biomarker for identifying patients who can better response to immunotherapies(8). We found that TTN, TP53, MUC16, CSMD3 and RYR2 composed the most commonly mutated gene signature lung squamous cell carcinoma. The observed increased frequency of TP53 mutations is related to poor prognosis in many types of tumor including breast cancer(9), lung cancer(10) and colon cancer(11). The mutation of MUC16 contributes to progression and in some malignant tumors(12). CSMD3 mutations are thought to be linked to a better prognosis(13). And there were studies found that RYR2 decreased the risk of breast cancer(14) and the expression of RYR2 is associated with prognosis and upcoming malignant conversion(15). The function of these mutated genes indicates that TMB has a certain relationship with the prognosis of patients with lung squamous cell carcinoma. High TMB showed better overall survival than low TMB group in multiple cancer types, including advanced gastric cancer(16),non-small-cell lung cancer (NSCLC) $(6,17)$, gastric carcinoma(18) and advanced esophagogastric cancer(19). In consistence with these findings, we found patients in high-TMB group showed better one- year overall survival.

Differentially expressed genes (DEGs) were identified and KEGG enrichment analysis showed that these DEGs mainly participated in lymphocyte activation, lymphocyte proliferation, leukocyte proliferation and mononuclear cell proliferation. We believed that these differential genes were closely related to immune function and even immune infiltrates in lung squamous cell carcinoma microenvironment. GSEA revealed that high-TMB group was associated with DNA replication and cell cycle process. While in the low TMB group, immune response pathways were enriched group. Therefore, we speculated that TMB may affect the development of tumors by changing the infiltration of immune cells and affecting the metabolism of cells.

Immune cells infiltration is closely correlated with the growth and invasion of tumor. The $T$ follicular helper cells (TFH), a subset of CD4 + T cells, play a pivotal role in humoral immunity(20). Several studies have considered infiltrating $T$ follicular helper cells may improve patients' survival and reduce immune suppression in many types of cancers, including breast cancer(21), colorectal cancer(22) and lung cancer(23). Macrophages are commonly grouped into the classically activated type 1 (M1) and the alternatively activated type 2 (M2). M1 macrophages can secrete pro-inflammatory cytokines, such as TNF-a, IL-1 $\beta$, IL- 6 and IL-12, as well as nitrogen/oxygen intermediates, which participate in tumoricidal 
activity and anti-tumor activity(24). M2 macrophages induced by IL-4 or IL-13 have immunomodulatory effects on anti-inflammatory and promote tumor activity(24). Jurgita Jackute found that high infiltration of M1 macrophages and low infiltration of M2 macrophages in tumor were associated with improved survival in NSCLC(25). And Saito et al. found that the infiltration of CD8 $+\mathrm{T}$ cells is associated with better survival(26).In our study, The T follicular helper cells infiltration, CD8 + T cell and M1 macrophages are higher in high- TMB groups which is correlated with positive survival outcomes.

Importantly, we also discussed the relationships between TMB and the expression of PD1/ PDL1.We found no association between TMB and PD-L1/PD1 expression, which was consistent with previous findings. Yu et al. reported that PD-L1 expression was not correlated with TMB in lung squamous cell carcinoma $(27,28)$.

However, there are some limitations: first, lack of basic experiment to validate the association between TMB and overall survival; second, lack of large clinical sample to verify the relationship between immune infiltrates and TMB. Relevant variants and big sample clinical trials are needed in the future.

\section{Conclusion}

Higher TMB correlated with better one-year survival outcomes. And TMB is association with the infiltration of immune cells in lung squamous cell carcinoma. We guess high TMB may improve the prognosis of patients with lung squamous cell carcinoma by changing the immune cell infiltration.

\section{Abbreviations}

\begin{tabular}{ll} 
TMB & Tumor Mutation Burden \\
\hline TCGA & The Cancer Genome Atlas \\
\hline NSCLC & Non-small cell lung cancer \\
\hline SNP & Single nucleotide polymorphism \\
\hline DEGs & Differentially expressed genes \\
\hline GSEA & Gene Set Enrichment Analysis \\
\hline FDR & False Discovery Rate
\end{tabular}

\section{Declarations}

\section{Ethics approval and consent to participate}

Not applicable

\section{Consent for publication}


Written informed consent for publication was obtained from all participants.

\section{Availability of data and materials}

The data used in this study can be downloaded from the TCGA database (https://portal.gdc.cancer.gov/).

\section{Competing interests}

The authors declare that they have no known competing financial interests or personal relationships that could have appeared to influence the work reported in this paper.

\section{Funding}

No funding was received

\section{Authors' contributions}

Dan yan proposed the conception and design of this research and analyzed and interpreted the data. Yi Chen collected data and performed preprocessing. Dan Yan were major contributors in writing the manuscript. All authors read and approved the final manuscript.

\section{Acknowledgments}

I would like to show my deepest gratitude to my colleague who has provided me with valuable guidance in every stage of the writing of this paper. Then I would thank all patients involved in these studies, especially the TCGA database.

\section{References}

1. Bray F, Ferlay J, Soerjomataram I, Siegel RL, Torre LA and Jemal A: Global cancer statistics 2018: GLOBOCAN estimates of incidence and mortality worldwide for 36 cancers in 185 countries. CA Cancer J Clin 68: 394-424, 2018.

2. Yip C, Blower PJ, Goh V, Landau DB and Cook GJ: Molecular imaging of hypoxia in non-small-cell lung cancer. Eur J Nucl Med Mol Imaging 42: 956-976, 2015.

3. Refolo MG, Lotesoriere C, Messa C, Caruso MG and D'Alessandro R: Integrated immune gene expression signature and molecular classification in gastric cancer: New insights. Journal of leukocyte biology2020.

4. Kikuchi T, Mimura $\mathrm{K}$, Ashizawa $\mathrm{M}$, et al.: Characterization of tumor-infiltrating immune cells in relation to microbiota in colorectal cancers. Cancer immunology, immunotherapy : CII 69: 23-32, 2020.

5. Liu X, Wu S, Yang Y, Zhao M, Zhu G and Hou Z: The prognostic landscape of tumor-infiltrating immune cell and immunomodulators in lung cancer. Biomed Pharmacother 95: 55-61, 2017. 
6. Goodman AM, Kato S, Bazhenova L, et al.: Tumor Mutational Burden as an Independent Predictor of Response to Immunotherapy in Diverse Cancers. Molecular cancer therapeutics 16: 2598-2608, 2017.

7. McFarland DC, Jutagir DR, Miller AH, Breitbart W, Nelson C and Rosenfeld B: Tumor Mutation Burden and Depression in Lung Cancer: Association With Inflammation. Journal of the National Comprehensive Cancer Network : JNCCN 18: 434-442, 2020.

8. Tafe LJ: Non-Small Cell Lung Cancer as a Precision Oncology Paradigm: Emerging Targets and Tumor Mutational Burden (TMB). Advances in anatomic pathology 27: 3-10, 2020.

9. Dobes P, Podhorec J, Coufal O, et al.: Influence of mutation type on prognostic and predictive values of TP53 status in primary breast cancer patients. Oncology reports 32: 1695-1702, 2014.

10. Mitsudomi T, Hamajima N, Ogawa M and Takahashi T: Prognostic significance of p53 alterations in patients with non-small cell lung cancer: a meta-analysis. Clinical cancer research : an official journal of the American Association for Cancer Research 6: 4055-4063, 2000.

11. Conlin A, Smith G, Carey FA, Wolf CR and Steele RJ: The prognostic significance of K-ras, p53, and APC mutations in colorectal carcinoma. Gut 54: 1283-1286, 2005.

12. Aithal $A$, Rauth $S$, Kshirsagar $P$, et al.: MUC16 as a novel target for cancer therapy. Expert opinion on therapeutic targets 22: 675-686, 2018.

13. La Fleur L, Falk-Sörqvist E, Smeds $P$, et al.: Mutation patterns in a population-based non-small cell lung cancer cohort and prognostic impact of concomitant mutations in KRAS and TP53 or STK11. Lung Cancer 130: 50-58, 2019.

14. Wei Y, Wang X, Zhang Z, et al.: Impact of NR5A2 and RYR2 3'UTR polymorphisms on the risk of breast cancer in a Chinese Han population. Breast cancer research and treatment 183: 1-8, 2020.

15. Schmitt K, Molfenter B, Laureano NK, et al.: Somatic mutations and promotor methylation of the ryanodine receptor 2 is a common event in the pathogenesis of head and neck cancer. International journal of cancer 145: 3299-3310, 2019.

16. Wang F, Wei XL, Wang FH, et al.: Safety, efficacy and tumor mutational burden as a biomarker of overall survival benefit in chemo-refractory gastric cancer treated with toripalimab, a PD-1 antibody in phase Ib/II clinical trial NCT02915432. Ann Oncol 30: 1479-1486, 2019.

17. Devarakonda S, Rotolo F, Tsao MS, et al.: Tumor Mutation Burden as a Biomarker in Resected NonSmall-Cell Lung Cancer. J Clin Oncol 36: 2995-3006, 2018.

18. Cho J, Ahn S, Son DS, et al.: Bridging genomics and phenomics of gastric carcinoma. International journal of cancer 145: 2407-2417, 2019.

19. Greally M, Chou JF, Chatila WK, et al.: Clinical and Molecular Predictors of Response to Immune Checkpoint Inhibitors in Patients with Advanced Esophagogastric Cancer. Clinical cancer research : an official journal of the American Association for Cancer Research 25: 6160-6169, 2019.

20. Eivazi S, Bagheri S, Hashemzadeh MS, et al.: Development of $T$ follicular helper cells and their role in disease and immune system. Biomed Pharmacother 84: 1668-1678, 2016. 
21. Gu-Trantien C, Loi S, Garaud S, et al.: CD4(+) follicular helper T cell infiltration predicts breast cancer survival. J Clin Invest 123: 2873-2892, 2013.

22. Wang Z, Wang Z, Diao Y, Qian X, Zhu N and Dong W: Circulating follicular helper T cells in Crohn's disease (CD) and CD-associated colorectal cancer. Tumour Biol 35: 9355-9359, 2014.

23. Xu F, Zhang H, Chen J, Lin L and Chen Y: Immune signature of $T$ follicular helper cells predicts clinical prognostic and therapeutic impact in lung squamous cell carcinoma. Int Immunopharmacol 81: 105932, 2020.

24. Jackaman C, Tomay F, Duong L, et al.: Aging and cancer: The role of macrophages and neutrophils. Ageing Res Rev 36: 105-116, 2017.

25. Jackute J, Zemaitis M, Pranys D, et al.: Distribution of M1 and M2 macrophages in tumor islets and stroma in relation to prognosis of non-small cell lung cancer. BMC Immunol 19: 3, 2018.

26. Tamminga M, Hiltermann TJN, Schuuring E, Timens W, Fehrmann RS and Groen HJ: Immune microenvironment composition in non-small cell lung cancer and its association with survival. Clinical \& translational immunology 9: e1142, 2020.

27. Jiang T, Shi J, Dong Z, et al.: Genomic landscape and its correlations with tumor mutational burden, PD-L1 expression, and immune cells infiltration in Chinese lung squamous cell carcinoma. Journal of hematology \& oncology 12: 75, 2019.

28. Yu H, Chen Z, Ballman KV, et al.: Correlation of PD-L1 Expression with Tumor Mutation Burden and Gene Signatures for Prognosis in Early-Stage Squamous Cell Lung Carcinoma. J Thorac Oncol 14: 25-36, 2019.

\section{Figures}


Variant Classification

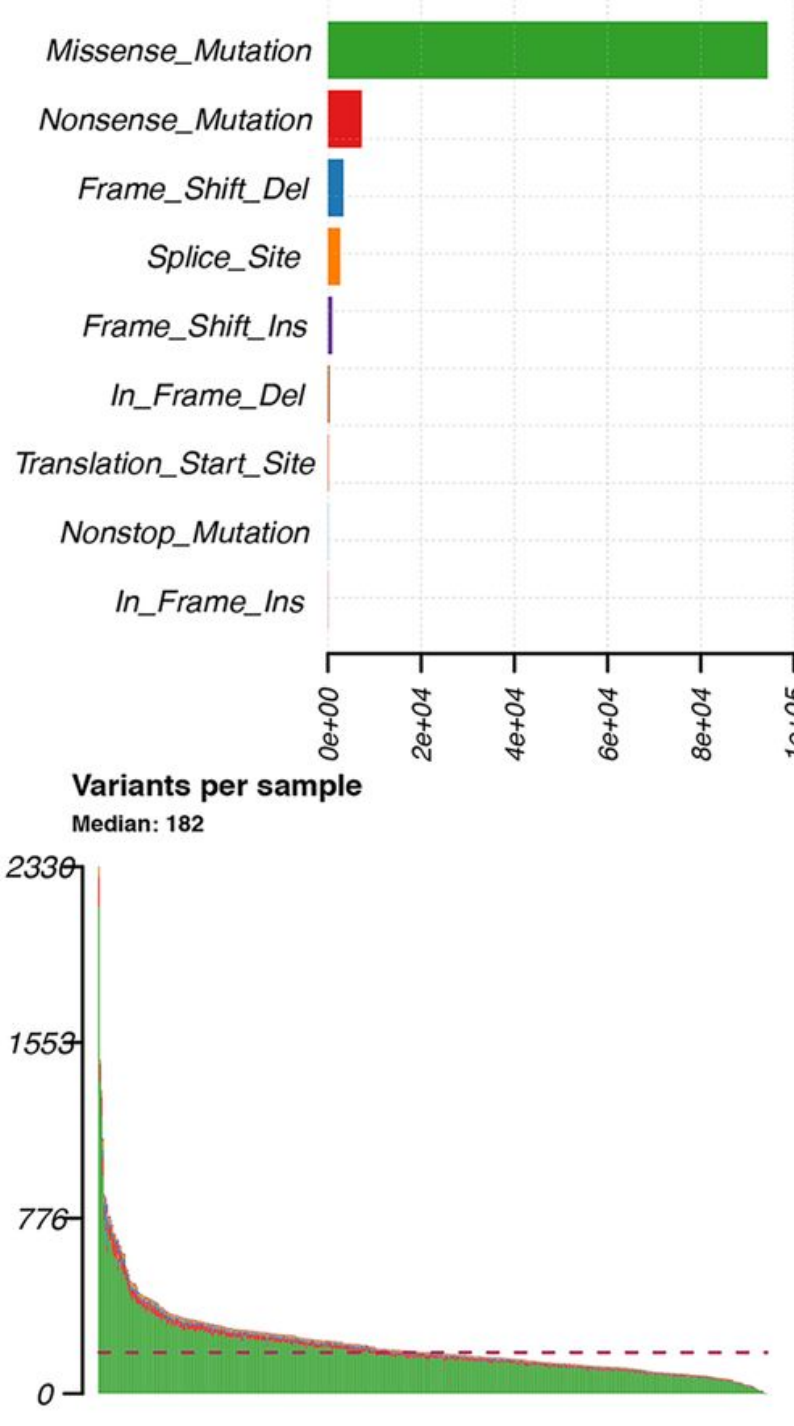

Variant Type

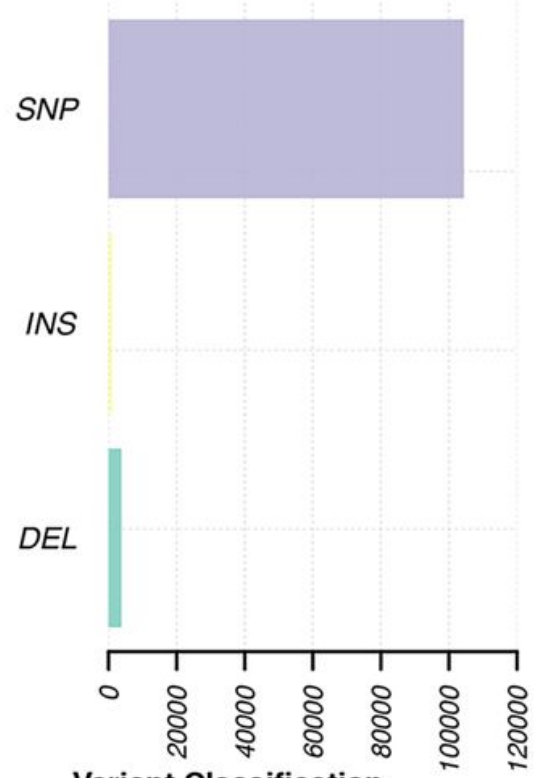

Variant Classification summary

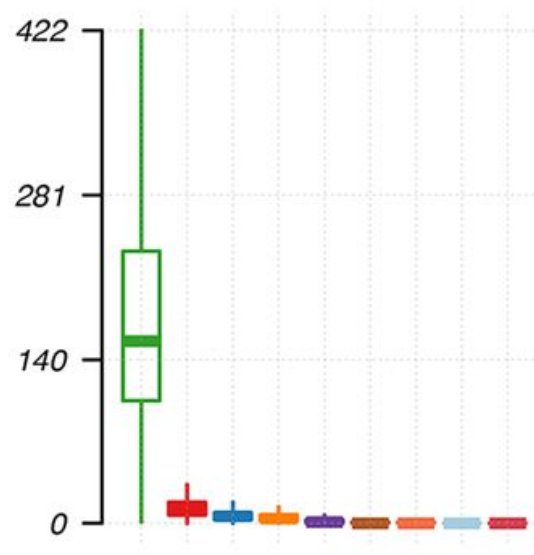

SNV Class

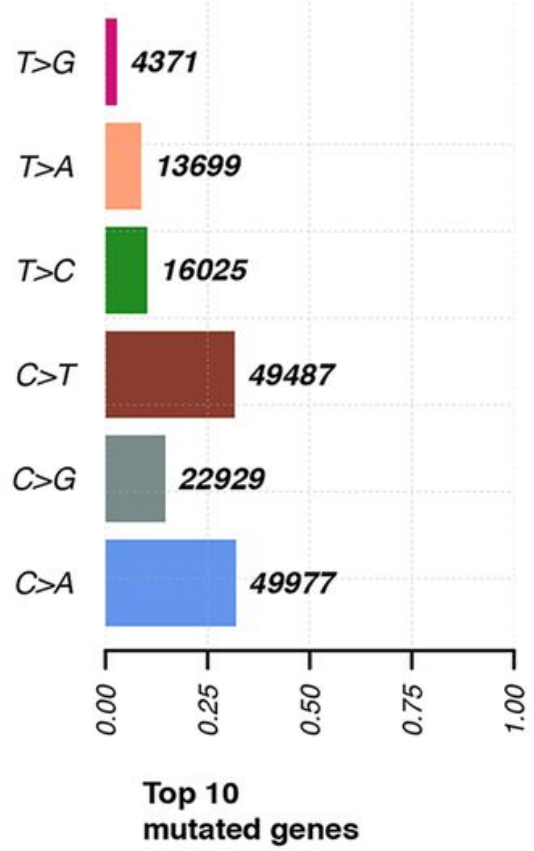

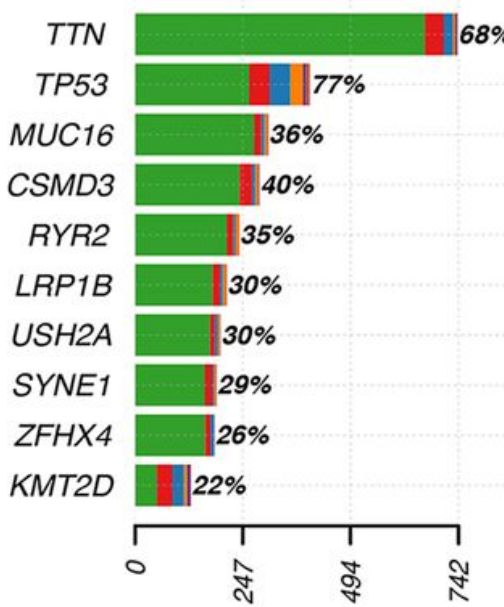

\section{Figure 1}

Summary of the mutation information with statistical calculations. 
Altered in $482(98.17 \%)$ of 491 samples.

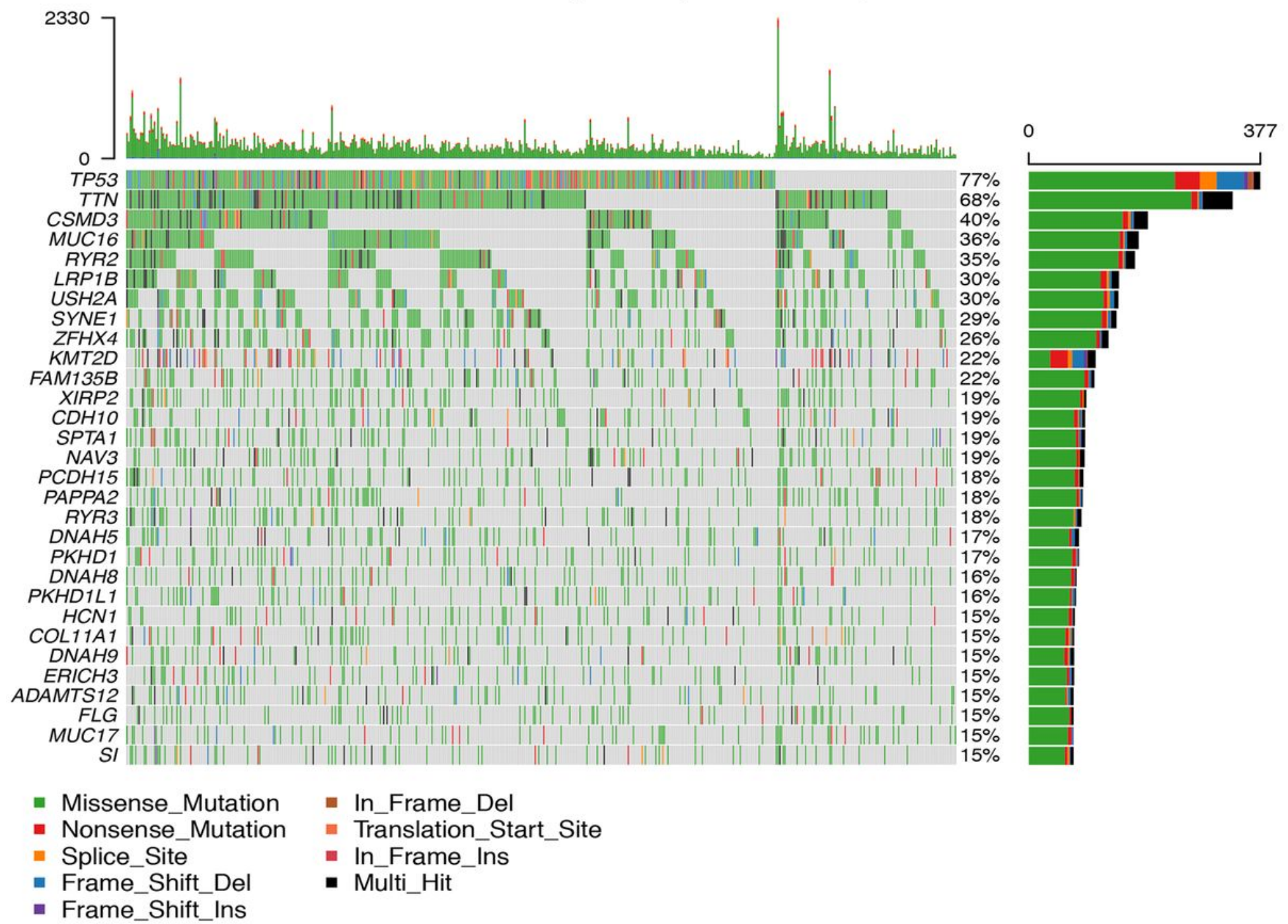

Figure 2

Mutation information of each gene in each sample was shown in the waterfall plot, in which various colors with annotations at the bottom represented the different mutation types. 


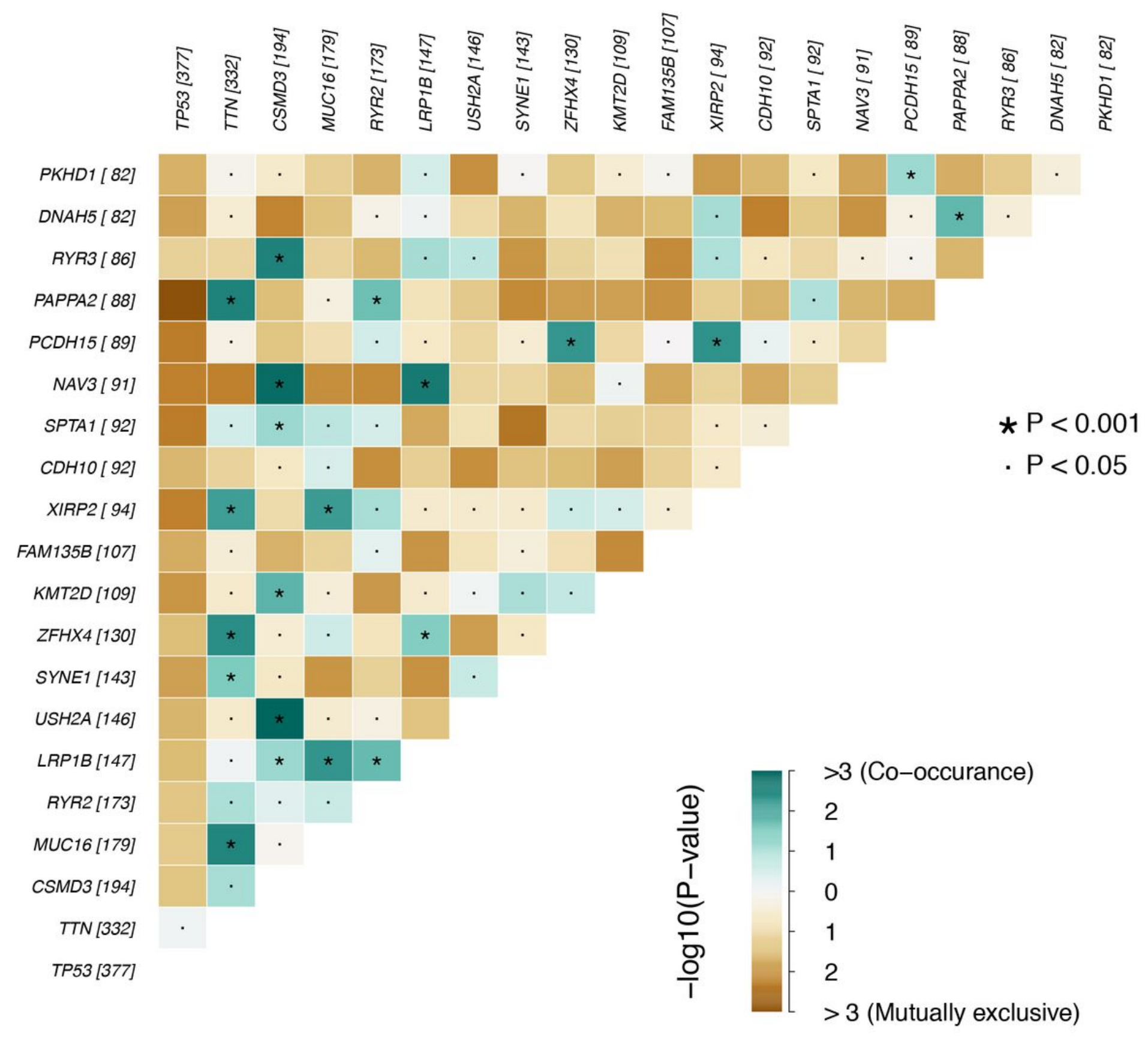

Figure 3

The coincident and exclusive associations across mutated genes. 
$\operatorname{TMB}(\mathrm{p}=0.006)$

A

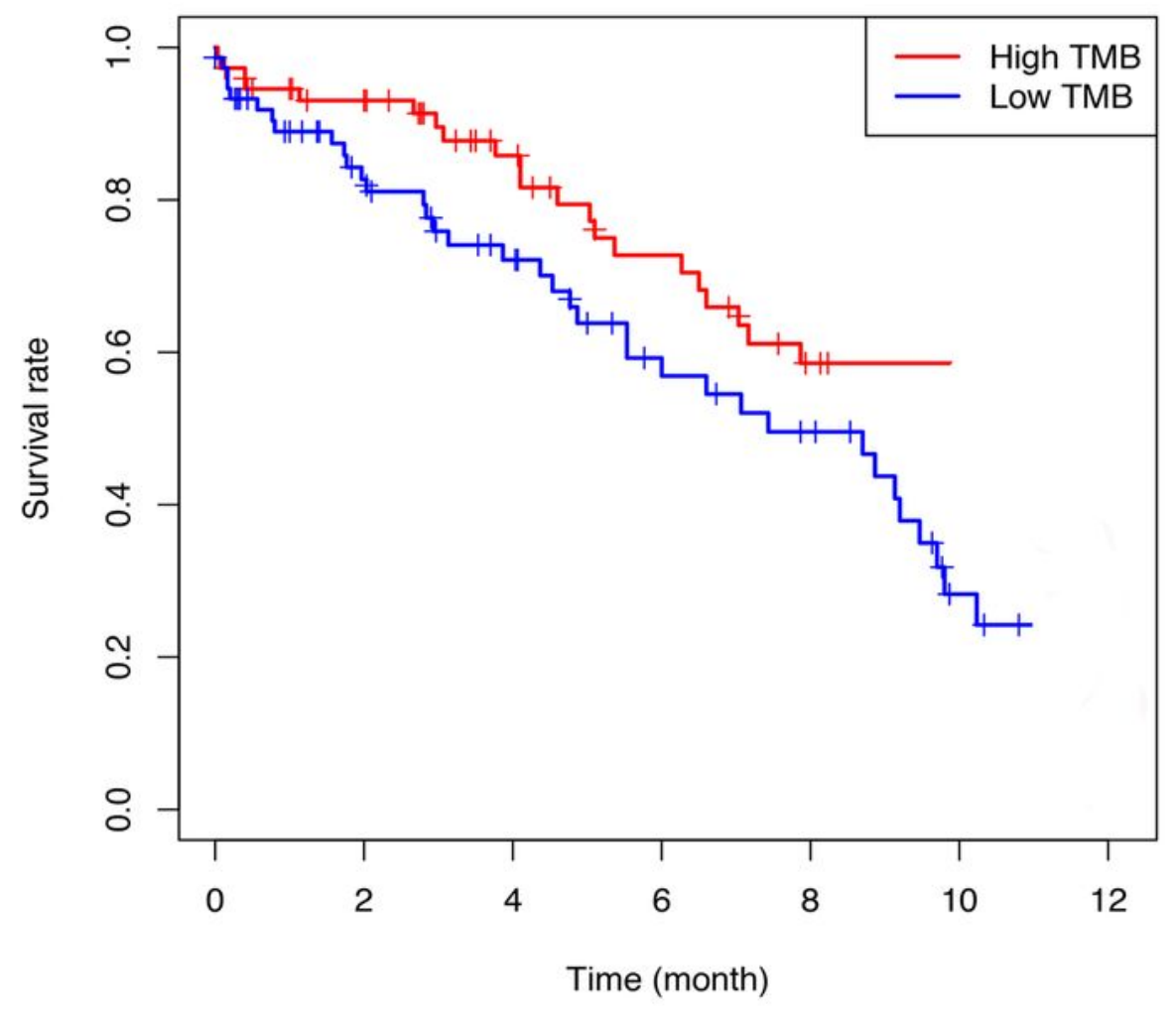

B TMB $(p=0.033)$

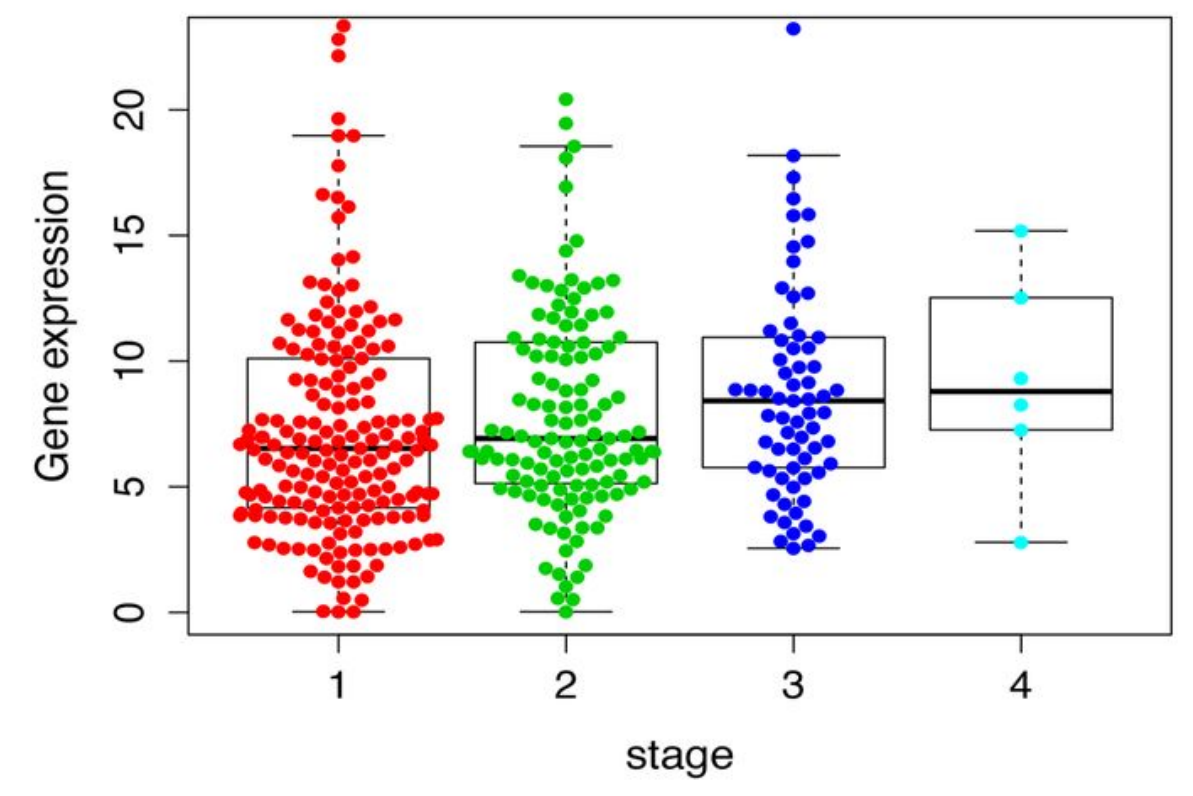

Figure 4

Prognosis of TMB and associations with risk clinical characteristics. (A) Higher TMB levels correlated with better one-year overall survival. (B) higher TMB level was associated with advanced stages. 

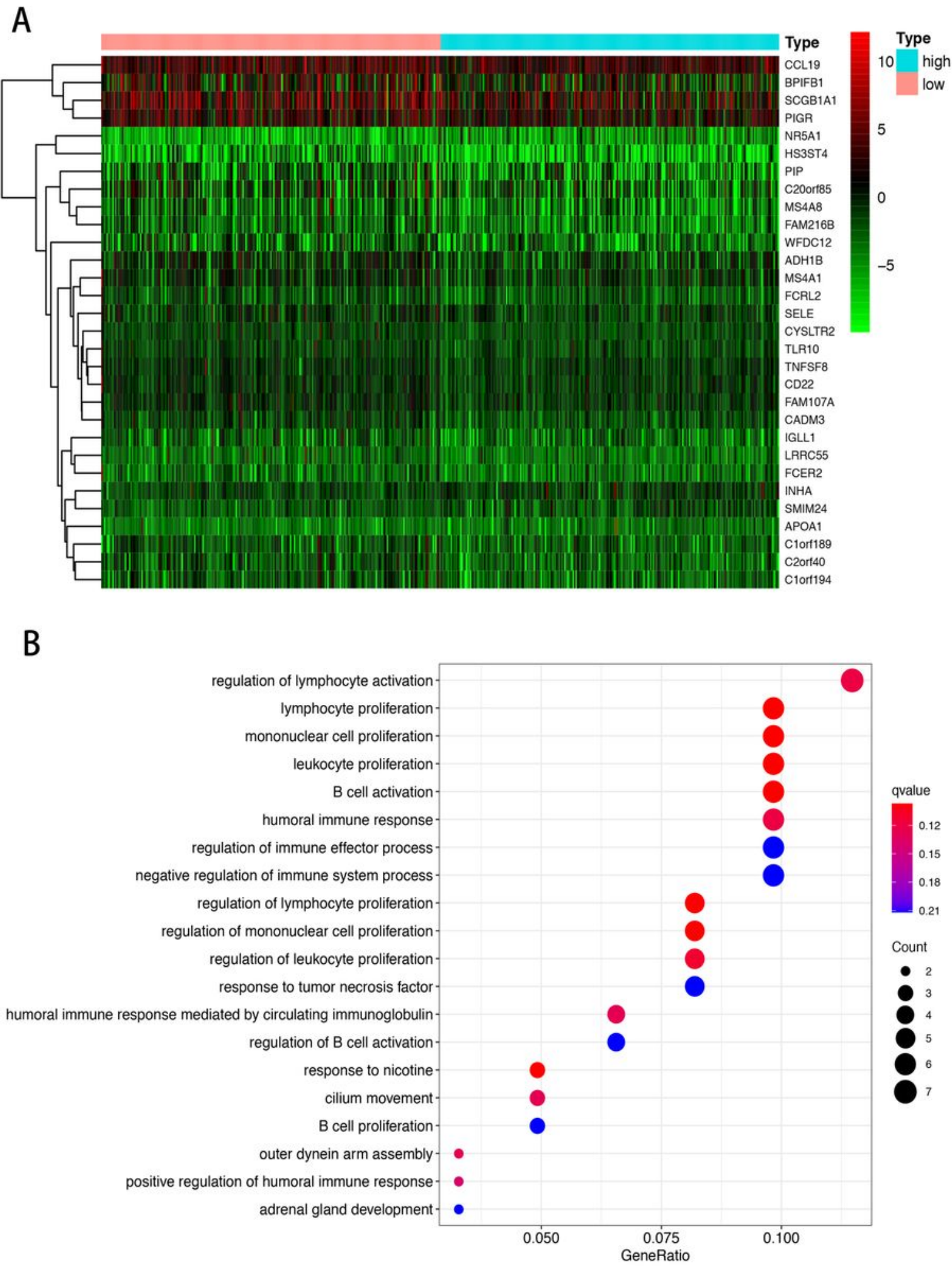

\section{Figure 5}

Comparisons of gene expression profiles in two different groups and KEGG enrichment analysis. (A) DEGs were shown in heatmap plot. (B) KEGG enrichment analysis 
Enrichment plot:
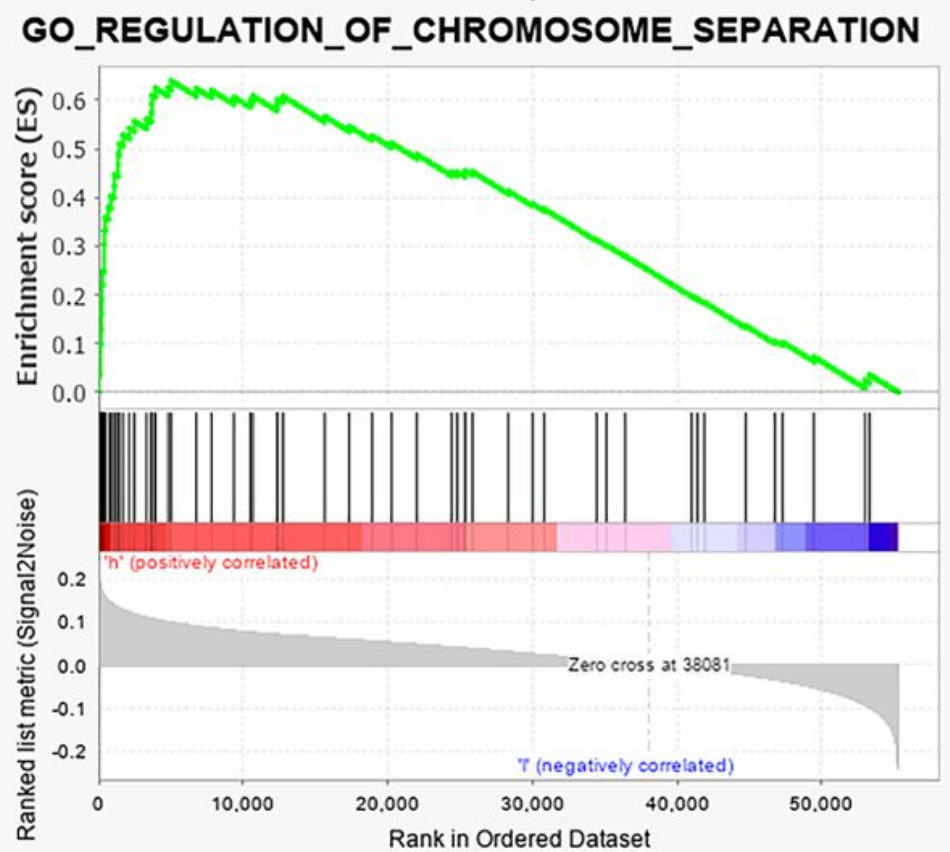

- Enrichment profile - Hits —Ranking metric scores

Enrichment plot: GO_DNA_POLYMERASE_COMPLEX

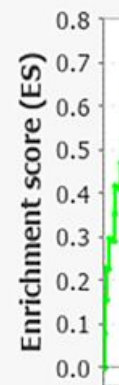

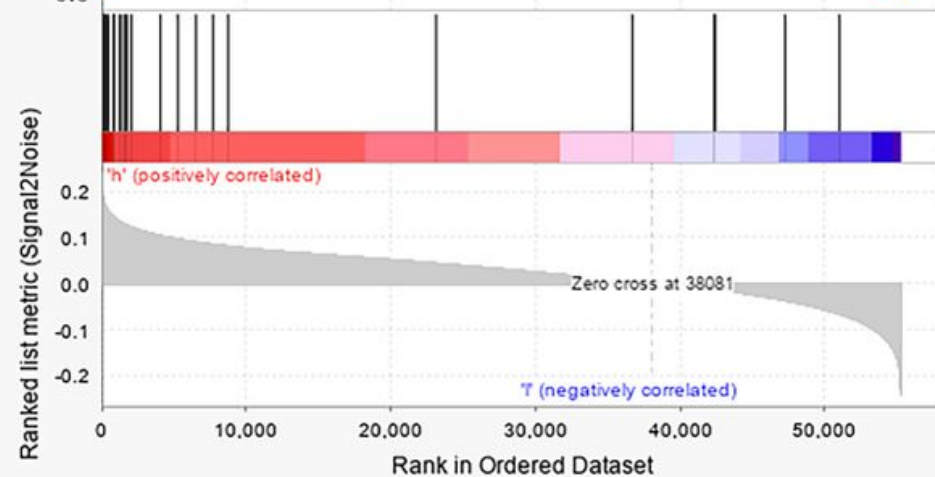

Rank in Ordered Dataset

- Enrichment profile — Hits — Ranking metric scores
Enrichment plot:

GO_MEIOSIS_I_CELL_CYCLE_PROCESS
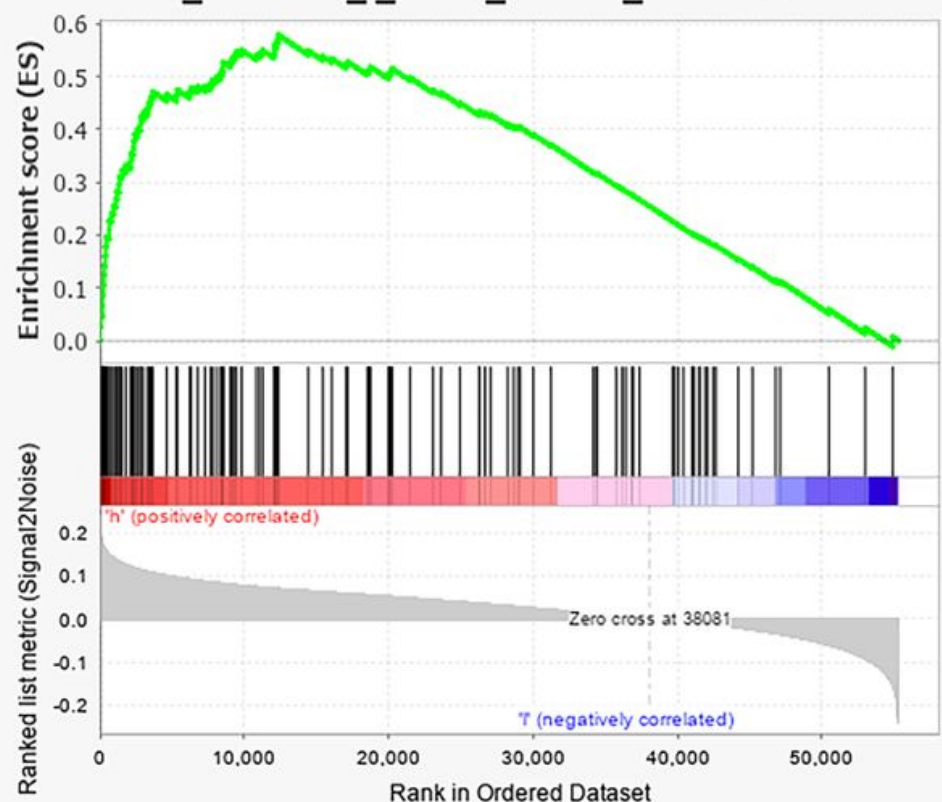

\section{- Enrichment profile — Hits — Ranking metric scores}

Enrichment plot: GO_DNA_REPLICATION_INITIATION

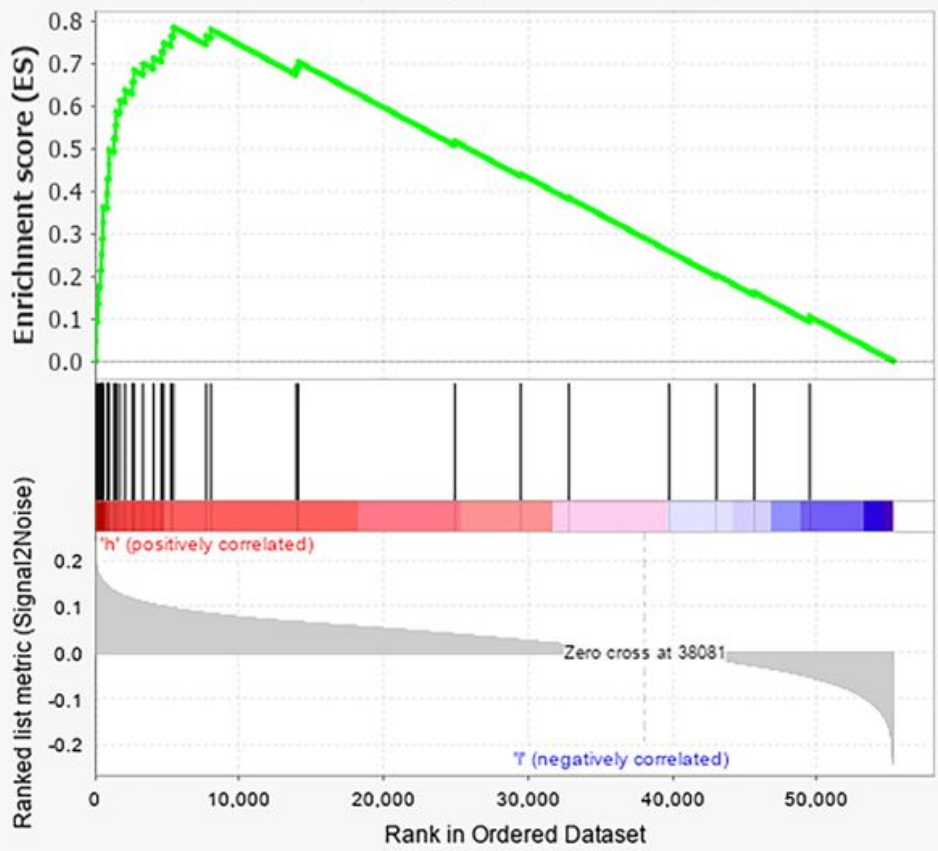

Enrichment profile — Hits

Ranking metric scores

\section{Figure 6}

TMB-related crosstalk by GSEA in high TMB group. 

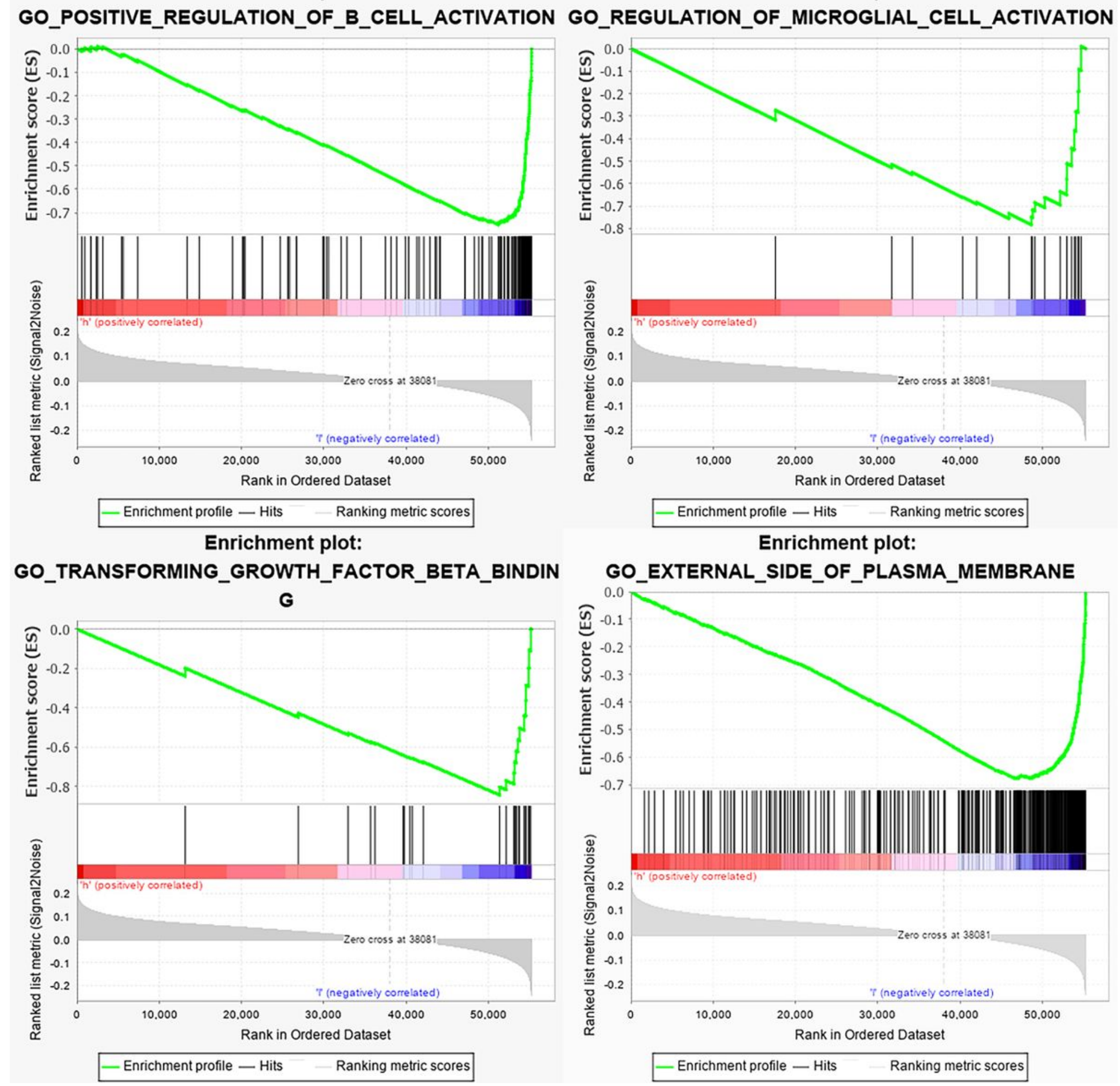

Figure 7

TMB-related crosstalk by GSEA in low TMB group 
A

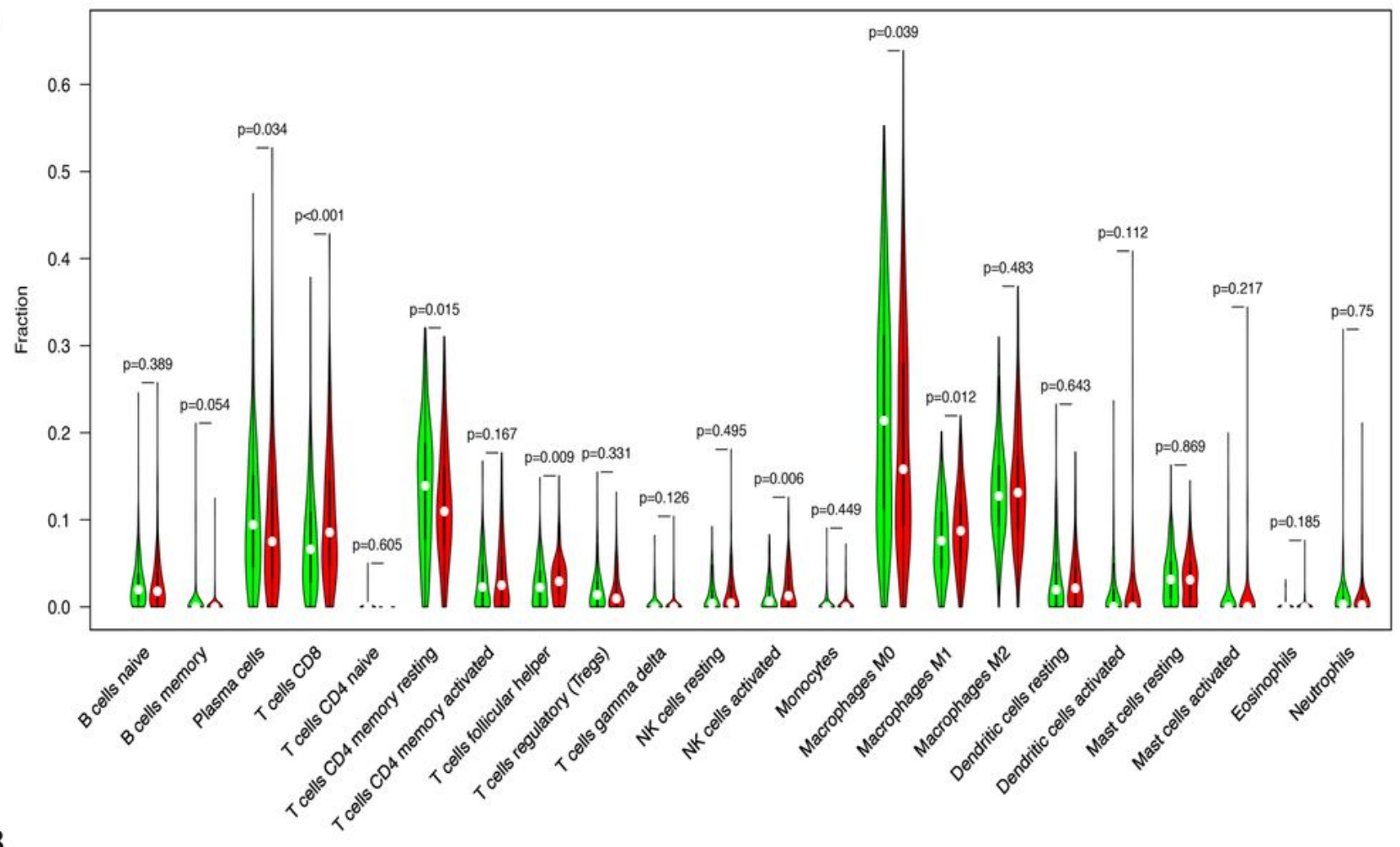

B

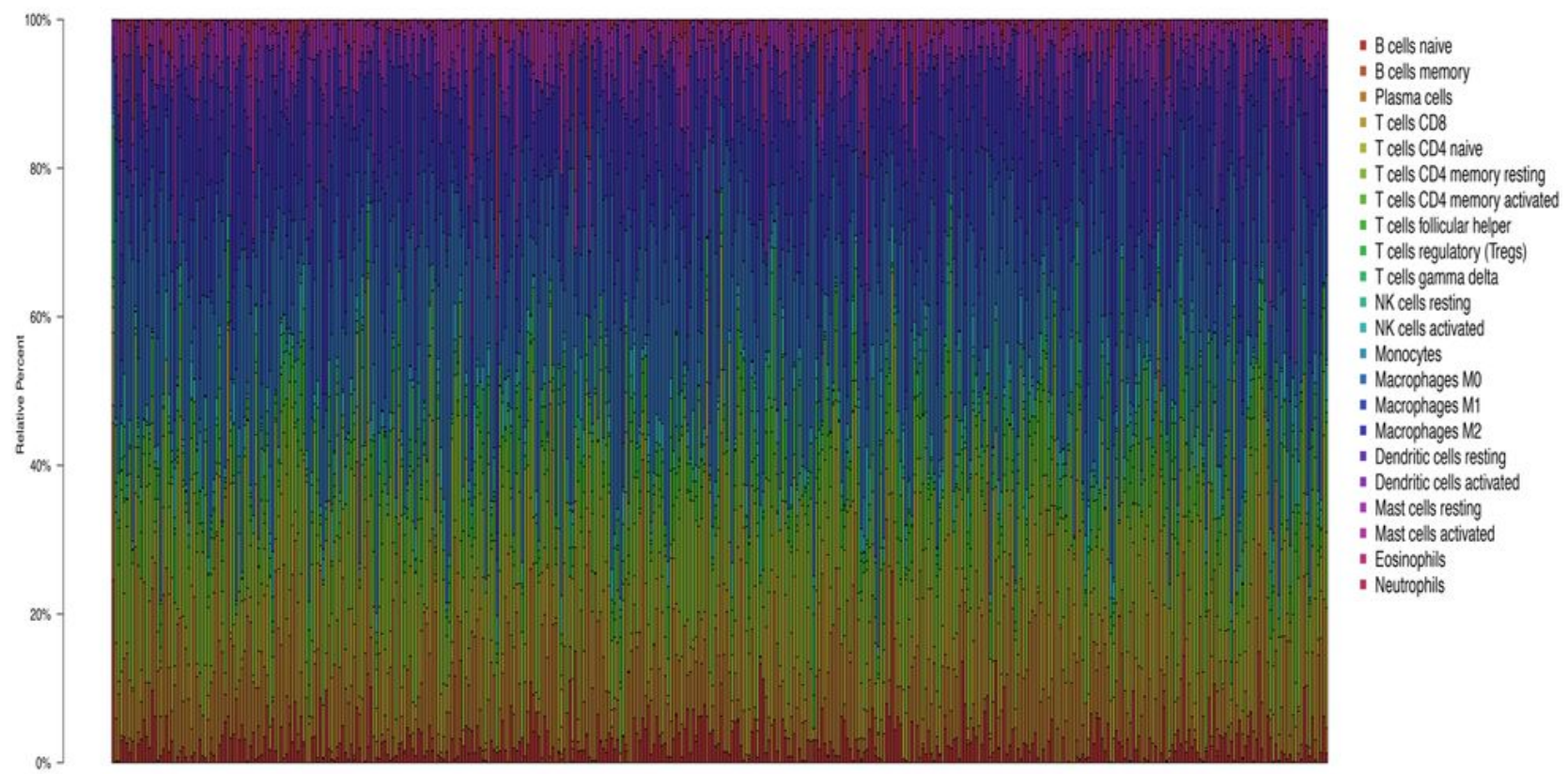

Figure 8

Comparisons of 22 immune cells infiltration between low- and high-TMB groups. 